\title{
Hodge Spectral Sequence on Compact Kähler Spaces
}

\author{
By \\ Takeo OHSAWA*
}

\section{Inptrodaction}

Let $X$ be a complex manifold of dimension $n$ and $E_{r}^{p, q}$ the Hodge spectral sequence on $X$. The following is fundamental in the study of algebraic varieties.

Theorem (W.V.D. Hodge [8]) If $X$ is a compact Kähler manifold, then

$$
\text { (H) }\left\{\begin{array}{l}
E_{1}^{p, q}=E_{\infty}^{p, q} \\
E_{1}^{p, q} \cong E_{1}^{p, q}
\end{array} \quad \text { for any } p \text { and } q\right. \text {. }
$$

In $1972, \mathbb{P}$. Deligne [3] succeeded in generalizing it for an arbitrary quasiprojective variety by analyzing a different spectral sequence. His so called mixed Hodge theory explains how the singular cohomology is composed of the analytic cohomology attached to the variety.

On the other hand, Grauert-Riemenschneider [7] and Fujiki [5] tried to understand the Hodge spectral sequence itself on pseudoconvex manifolds. Inspired by these works, the author [11] could show that $(H)$ is valid for the range $p+q \geq n+r$ on any "very strongly $r$-convex" Kähler manifold of dimension $n$. The crucial point was to establish an isomorphism between the ordinary cohomology and the $L^{2}$ cohomology with respect to a certain complete Kähler metric on pseudoconvex domains.

Since it has long been known that for any projective variety over $\mathbb{C}$ the complement of the singular locus admits a complete $\mathbb{K a ̈ h l e r}$ metric (Grauert [6]), it is natural to ask for a reasonable extension of [11] in such a case.

The purpose of the present paper is to show the following in this spirit.

Theorem $\mathbb{1}$ Let $X$ be a compact Kähler space of pure dimension $n$ whose

Received July 15, 1986.

* Research Institute for Mathematical Sciences, Kyoio University, Kyoto 606, Japan. 
singular points are isolated, and let $X^{*}$ be the complement of the singular points. Then $(H)$ holds on $X^{*}$ for the range $p+q<n-1$.

Note that the range is optimal since $\operatorname{dim} H^{0, n-1}\left(X^{*}\right)=\infty$ if $X^{*} \neq X$, where $H^{0, n-1}$ denotes the Dolbeault cohomology of type $(n-1,0)$.

We shall also give a partial answer to a question of Cheeger-GoreskiMacPherson [2] by showing the following:

Theorem 2 Under the situation of Theorem 1,

$$
\begin{cases}H^{r}\left(X^{*}\right) \cong H_{(2)}^{r}\left(X^{*}\right) & \text { if } r<n-1 \\ H^{p, q}\left(X^{*}\right) \cong H_{(2)}^{p, q}\left(X^{*}\right) & \text { if } p+q<n-1\end{cases}
$$

and

$$
\begin{aligned}
& H_{0}^{r}\left(X^{*}\right) \cong H_{(2)}^{r}\left(X^{*}\right) \quad \text { if } \quad r>n+1 \\
& H_{0}^{p, q}\left(X^{*}\right) \cong H_{(2)}^{p, q}\left(X^{*}\right) \text { if } p+q>n+1 .
\end{aligned}
$$

Here. $H, H_{0}$ and $H_{(2)}$ denote respectively the ordinary cohomology, the cohomology with compact support, and the $L^{2}$ cohomology.

Note that the duality between $H_{(2)}^{r}$ and $H_{(2)}^{2 n-r}$ is not obvious since the metric on $X^{*}$ is not complete as long as $X^{*} \neq X$.

Since the intersection cohomology $I H^{r}(X)$ is isomorphic to $H^{r}\left(X^{*}\right)$ if $r<n$ and isomorphic to $H_{0}^{r}\left(X^{*}\right)$ if $r>n$, Theorem 2 implies the following.

$$
\text { Corollary } \quad I H^{r}(X) \cong H_{(2)}^{r}\left(X^{*}\right) \quad \text { if } \quad r \neq n, n \pm 1 .
$$

Cheeger-Goreski-MacPherson conjectured that the above isomorphism is valid for any degree, and in some special cases it has been verified (cf. [2], [10] and [12]).

The manuscript was written during the author's stay in the University of Göttingen during the summer of '86. He thanks to the institute of mathematics for the hospitality.

\section{$\S 1$. Preliminaries}

Let $\left(M, d s^{2}\right)$ be a complete Hermitian manifold of dimension $n$. We put

$$
\begin{aligned}
& L_{(2)}\left(=L_{(2)}(M)\right):=\{\text { square integrable complex } \\
&\text { differential forms on } M\} . \\
& L_{(2)}^{r}\left(=L_{(2)}^{r}(M)\right):=\left\{f \in L_{(2)} ; \operatorname{deg} f=r\right\} .
\end{aligned}
$$




$$
L_{(2)}^{p, q}\left(=L_{(2)}^{p, q}(M)\right):=\left\{f \in L_{(2)} ; f \text { is of type }(p, q)\right\} .
$$

The norms and the inner products in $L_{(2)}$ shall be denoted by \|\|$\left(=\|\|_{M}\right)$ and $(),\left(=(,)_{M}\right)$, respectively. The exterior differentiations $d, \bar{\partial}$ and $\partial$ are regarded as densely defined closed linear operators on $L_{(2)}$ whose domains of definition are given by

$$
\text { Dom } d:=\left\{f \in L_{(2)} ; d f \in L_{(2)}\right\} \text {, etc . }
$$

Here the differentiation is in distribution sense.

Defimition

$$
\begin{aligned}
& H_{(2)}\left(=H_{(2)}(M)\right):=\operatorname{Ker} d / \operatorname{Im} d \\
& H_{(2)}^{r}\left(=H_{(2)}^{r}(M)\right):=\operatorname{Ker} d \cap L_{(2)}^{r} / \operatorname{Im} d \cap L_{(2)}^{r} . \\
& H_{(2)}^{p, q}\left(=H_{(2)}^{p, q}(M)\right):=\operatorname{Ker} \bar{\partial} \cap L_{(2)}^{p, q} / \operatorname{Im} \bar{\partial} \cap L_{(2)}^{p, q} .
\end{aligned}
$$

We denote by $d^{*}$ and $\bar{\partial}^{*}$ the adjoints of $d$ and $\bar{\partial}$, respectively. Note that $H_{(2)} \cong \operatorname{Ker} d \cap \operatorname{Ker} d^{*}$ (resp. $\left.H_{(2)}^{p, q} \cong \operatorname{Ker} \bar{\partial} \cap \operatorname{Ker} \bar{\partial}^{*} \cap L_{(2)}^{b, q}\right)$ if and only if $\operatorname{Im} d$ is closed (resp. $\operatorname{Im} \bar{\partial} \cap L_{(2)}^{p, q}$ is closed). $H_{(2)}$ are called $L^{2}$ cohomologies of $M$. For any family of supports $\Phi, L^{2}$ cohomologies with supports in $\Phi$ are also defined similarly as above.

The following is first due to H. Donnelly and C. Fefferman, but the proof below is different from theirs.

Theorem 1.1 (cf. [4]) Suppose that there exists a $C^{\infty}$ real valued function $F$ on $M$ such that the fundamental form of $d s^{2}$ is $i \partial \bar{\partial} F$ and that $|\partial F|_{\infty}(:=\sup$ $|\partial F|)<\infty$. Then, for any $u \in \operatorname{Ker} d \cap L_{(2)}^{r}$ with $r \neq n$, there exists $a v \in \mathbb{D o m} d$ $\cap L_{(2)}^{r-1}$ such that $d v=u$ and $\|v\| \leq 2|\partial F|_{\infty}\|u\|$. Similarly, if $p+q \neq n$, then for any $u \in \operatorname{Ker} \bar{\partial} \cap L_{(2)}^{p, q}$ there exists a $v \in \operatorname{Dom} \bar{\partial} \cap L_{(2)}^{p, q-1}$ such that $\bar{\partial} v=u$ and $\|v\| \leq(1+\sqrt{2})|\partial F|_{\infty}\|u\|$. In particular,

$$
\left\{\begin{array}{lll}
H_{(2)}^{r}=0 & \text { if } & r \neq n, \\
H_{(2)}^{p, q}=0 & \text { if } & p+q \neq n .
\end{array}\right.
$$

Proof. The assertions are equivalent to that

$$
\|u\| \leq 2|\partial F|_{\infty}\left\|d^{*} u\right\|, \quad \text { for any } u \in \operatorname{Ker} d \cap \operatorname{Dom} d^{*} \cap L_{(2)}^{r}
$$

and

$$
\|u\| \leq(1+\sqrt{2})|\partial F|_{\infty}\left\|\bar{\partial}^{*} u\right\|, \quad \text { for any } u \in \operatorname{Ker} \bar{\partial} \cap \operatorname{Dom} \bar{\partial}^{*} \cap L_{(2)}^{p, q},
$$

respectively (cf. [9]). They are proved as follows: 
For any differential form $\theta$, let $e(\theta)$ be the multiplication by $\theta$ from the left. Then we have the following formula.

$$
\left[\bar{\partial}, e(\bar{\partial} F)^{*}\right]+\left[\partial^{*}, e(\partial F)\right]=\left[e(i \partial \bar{\partial} F), e(i \partial \bar{\partial} F)^{*}\right] \text {. }
$$

Here $[$,$] denotes the commutator with weight (i.e., [S, T]:=S \circ T-(-1)^{\operatorname{deg} S \operatorname{deg} T}$ $T \circ S)$ and * denotes the adjoint.

In fact, with respect to the operator $\Lambda:=e(i \partial \bar{\partial} F)^{*}$ we have $[\bar{\partial}, \Lambda]=i \partial^{*}$ and $[e(\partial F), \Lambda]=i e(\bar{\partial} F)^{*}$ (cf. [12]). Therefore

$$
\begin{aligned}
& {[\bar{\partial},}\left.e(\bar{\partial} F)^{*}\right]=\bar{\partial} e(\bar{\partial} F)^{*}+e(\bar{\partial} F)^{*} \bar{\partial} \\
& \quad=-i \bar{\partial}[e(\partial F), \Lambda]-i[e(\partial F), \Lambda] \bar{\partial} \\
& \quad=[e(i \partial \bar{\partial} F), \Lambda]+i e(\partial F)[\bar{\partial}, \Lambda]+i[\bar{\partial}, \Lambda] e(\partial F) \\
& \quad=[e(i \partial \bar{\partial} F), \Lambda]-\left[e(\partial F), \partial^{*}\right] .
\end{aligned}
$$

Hence, for any compactly supported $C^{\infty} r$-form $u$,

$$
\begin{aligned}
& ([e(i \partial \bar{\partial} F), \Lambda] u, u) \\
& \quad \leq|\partial F|_{\infty}\|u\|(\|\bar{\partial} u\|+\|\bar{\partial} * u\|+\|\partial u\|+\|\partial * u\|) .
\end{aligned}
$$

Since the metric is Kählerian, we have

$$
\begin{aligned}
& \|\bar{\partial} u\|^{2}+\|\bar{\partial} * u\|^{2}=\|\partial u\|^{2}+\left\|\partial^{*} u\right\|^{2} \\
& =\frac{1}{2}\left(\|d u\|^{2}+\left\|d^{*} u\right\|^{2}\right) \quad(\text { cf. [14]) }
\end{aligned}
$$

On the other hand, $[e(i \partial \bar{\partial} F), \Lambda] u=(r-n) u$. Thus we obtain

$$
\|u\| \leq 2|\partial F|_{\infty}\left(\|d u\|^{2}+\left\|d^{*} u\right\|^{2}\right)^{1 / 2}
$$

and

$$
\|u\| \leq(1+\sqrt{2})|\partial F|_{\infty}(\|\bar{\partial} u\|+\|\bar{\partial} * u\|),
$$

if $r \neq n$.

Since the metric $d s^{2}$ is complete, the required estimate follows from the above (cf. [13]).

\section{§2. A Poincaré-Dollbeault Lemma}

Let $X$ be a complex analytic space of pure dimension $n$. In what follows the nonsingular part of $X$ will be denoted by $X^{*}$. Suppose that $o$ is an isolated singular point of $X$. Then we have a holomorphic embedding of the 
germ $(X, \underline{o}) \hookrightarrow\left(\mathbb{C}^{N}, O\right)$. We fix in the followings a holomorphic coordinate $z\left(=\left(z_{1}, \cdots, z_{N}\right)\right)$ of $\mathbb{C}^{N}$ and the euclidean norm $|z|$ of $z$. We put $B_{c}^{*}:=\{z$; $0<|z|<c\}$ and $X_{c}^{*}\left(=X_{o, c}^{*}\right):=X \cap B_{c}^{*}(c$ sufficiently small). As a candidate of the potential $F$ in Theorem 1.1, we pul

$$
F_{c}(z)\left(=F_{c, o}(z)\right):=-\log \log (c /|z|) .
$$

Proposition 2.1 The length of $\partial\left(F_{c} \mid X_{c}^{*}\right)$ with respect to the metric $2 \partial \bar{\partial}$ $\left(F_{c} \mid X_{c}^{*}\right)$ is bounded.

Proof. On $B_{c}^{*}$ we have

$$
\partial F_{c}=\frac{-\partial \log |z|}{\log (c /|z|)}
$$

and

$$
\partial \bar{\partial} F_{c} \geq \frac{\partial \log |z| \bar{\partial} \log |z|}{\log ^{2}(c /|z|)} .
$$

Hence $\left|\partial\left(F_{c} \mid X_{c}^{*}\right)\right| \leq 1$.

In what follows we fix $c$ and regard $X_{b}^{*}$ for $b \leq c$ as a Kähler manifold with metric $2 \partial \bar{\partial}\left(F_{b} \mid X_{b}^{*}\right)$. Moreover $c$ is fixed so that $\partial \bar{X}_{b}^{*}$ is compact for all $b \leq c$. It is clear from (3) that $X_{b}^{*}$ are then complete Kähler manifolds.

Combining (1) in Theorem 1.1 and Proposition 2.1 we obtain the following:

Proposition 2.2 For any $b \leq c$,

$$
\left\{\begin{array}{lll}
H_{(2)}^{r}\left(X_{b}^{*}\right)=0 & \text { if } & r \neq n, \\
H_{(2)}^{p, q}\left(X_{b}^{*}\right)=0 & \text { if } & p+q \neq n .
\end{array}\right.
$$

The following observation was already made in [10], but we shall repeat the proof because of the completeness.

Lemma 2.3 Let $r>n$ and $u \in L_{(2)}^{r}\left(X_{c}^{*}\right)$. Then, $u \mid X_{b}^{*} \in L_{(2)}^{r}\left(X_{b}^{*}\right)$, for any $b \leq c$.

Proof. Since

$$
\partial \bar{\partial} F_{b}=\frac{\partial \bar{\partial} \log |z|}{\log (b /|z|)}+\frac{\partial \log |z| \bar{\partial} \log |z|}{\log ^{2}(b /|z|)},
$$

for any $b$, the eigenvalues $\lambda_{1} \leq \cdots \leq \lambda_{N}$ of $\partial \bar{\partial} F_{b}$ measured by $\partial \bar{\partial} F_{c}$ are given by

$$
\lambda_{j}=\frac{\log (c /|z|)}{\log (b /|z|)}, \quad \text { for } \quad 1 \leq j \leq N-1
$$


and

$$
\lambda_{N}=\frac{\log ^{2}(c /|z|)}{\log ^{2}(b /|z|)}
$$

Let $\mu_{1} \leq \cdots \leq \mu_{n}$ be the eigenvalues of $\partial \bar{\partial}\left(F_{b} \mid X_{b}^{*}\right)$ measured by $\partial \bar{\partial}\left(F_{c} \mid X_{c}^{*}\right)$. Then, by Courant's minimax principle,

$$
\mu_{j}=\frac{\log (c /|z|)}{\log (b /|z|)}, \quad \text { for } \quad 1 \leq j \leq n-1
$$

and

$$
\frac{\log (c /|z|)}{\log (b /|z|)} \leq \mu_{n} \leq \frac{\log ^{2}(c /|z|)}{\log ^{2}(b /|z|)} .
$$

Now it is easy to see that $\left\|u \mid X_{b}^{*}\right\|_{X^{*}} \leq\|u\|_{X_{c}^{*}}$, for any $u \in L_{(2)}^{r}\left(X_{c}^{*}\right)$ with $r>n$.

On the opposite side $r<n$ we have the following, which will be used to prove Theorem 2.

Lemma 2.4 Let $b<c$ and $u \in L_{(2)}^{r}\left(X_{b}^{*}\right)$ with $r<n$. Let $\tilde{u}$ be a form in $L_{(2)}^{r}\left(X_{c}^{*}\right)$ defined by $\tilde{u}:=u$ on $X_{b}^{*}$ and $\tilde{u}:=0$ on $X_{c}^{*} \backslash X_{b}^{*}$. Then $\tilde{u} \in L_{(2)}^{r}\left(X_{c}^{*}\right)$. Moreover if $r<n-1$, then $\tilde{u} \in \operatorname{Dom} d$ (resp. $\tilde{u} \in \operatorname{Dom} \bar{\partial}$ ) if $u \in \operatorname{Dom} d$ (resp. $u \in \operatorname{Dom} \bar{\partial}$ ).

Proof. The first part is proved similarly as in the proof of Lemma 2.3. The latter part follows from the first part and (3). In fact, let $\chi: \mathbb{R} \rightarrow \mathbb{R}$ be a $C^{\infty}$ function such that $\chi \equiv 1$ on $(-\infty,-2)$ and $\chi \equiv 0$ on $(-1, \infty)$. Then, if $u \in$ Dom $d$ (resp. Dom $\bar{\partial}$ ), the sequence $\{\chi(k(|z|-b)) u\}_{k=1}^{\infty}$ is convergent on $X_{c}^{*}$ with respect to the graph norm of $d$ (resp. the graph norm of $\bar{\partial}$ ), if $r$ $<n-1$.

Let $\Phi$ be a family of closed subsets of $X_{c}^{*}$ defined by $\Phi:=\left\{K \subset X_{c}^{*}\right.$; $K \cap \overline{X_{c / 2}^{*}}$ is compact\}.

Then the following is an immediate consequence of Proposition 2.2 and Lemma 2.4 .

Theorem 2.5 The images of the following natural homomorphisms are zero.

$$
\begin{array}{ll}
H_{0}^{r}\left(X_{c}^{*}\right) \rightarrow H_{\Phi}^{r}\left(X_{c}^{*}\right), & \text { for } r<n, \\
H_{0}^{p, q}\left(X_{c}^{*}\right) \rightarrow H_{\Phi}^{p, q}\left(X_{c}^{*}\right), & \text { for } \quad p+q<n .
\end{array}
$$

Here $H_{\Phi}$ denotes the cohomology with supports in $\Phi$. 
Theorem 2.5 is not used to prove Theorem 1 and Theorem 2, buc it may have some application in the theory of isolated singularities.

\section{§3。 Prool of Theorem 11}

Let $X$ be a complex space of dimension $n$. For any isolated singular point $\underline{o} \in X$ we shall freely use the notations $X_{c}, F_{c}$, etc. in $\S 2 . \quad X$ is called a

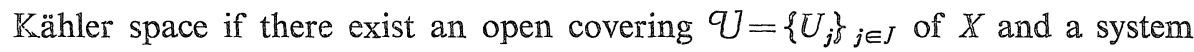
of $C^{\infty}$ strictly plurisubharmonic functions $\varphi_{j}$, each $\varphi_{j}$ being defined on $U_{j} \in \mathcal{U}$, such that $\varphi_{j}-\varphi_{k}$ is pluriharmonic on $U_{j} \cap U_{k^{*}}$. Given such a system of functions, $\left\{\partial \bar{\partial} \varphi_{j}\right\}_{j \in J}$ defines a Kähler metric on $X^{*}$. Clearly, this metric is locally quasi-isometric to those induced from the euclidean one by embedding $\mathbb{X}$ locally into $\mathbb{C}^{N}$.

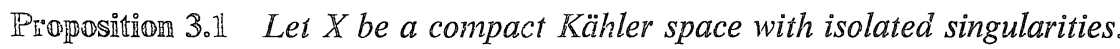
Then $X^{*}$ admits a complete Kähler metric which is quasi-isometric to $\partial \bar{\partial} F_{\underline{o}, c}$ lon $X_{o, c / 2}^{*}$ for each singular point $\underline{0}$.

Proof. Let $\underline{o}_{\nu}(\nu=1, \cdots, m)$ be the singular points of $X$. We choose $c$ so that $X_{o_{\nu}, c}^{*}$ are mutually disjoint regarded as subsets of $X$. Let $\rho_{\nu}$ be $\mathbb{C}^{\infty}$ functions on $X^{*}$ such that $\rho_{\nu}=1$ on $X_{\underline{o}, c / 2}^{*}$ and $\rho_{\nu}=0$ on $X^{*} \backslash X_{\underline{\alpha}, c}^{*}$. Then, for $A \gg 0$,

$$
\sum_{\nu=1}^{m} \partial \bar{\partial}\left(\rho_{\nu} \mathbb{F}_{\underline{o}_{\nu} c}\right)+A \partial \bar{\partial} \varphi_{j}
$$

gives a complete $\mathbb{K}$ ähler metric with the required property.

Proof of Theorem 1 Once for all we regard $X^{*}$ as a complete $\mathbb{K} a ̈ h l e r$ manifold with a metric such as in Proposition 3.1. Since $\mathbb{H}_{(2)}^{r}\left(X^{*}\right)=\underset{p+q=r}{\bigoplus}$ $H_{(2)}^{p, q}\left(X^{*}\right)$ and $H_{(2)}^{p, q}\left(X^{*}\right)=\overline{H_{(2)}^{q, p}\left(X^{*}\right)}$, it suffices to show that

$$
\begin{cases}\mathbb{H}_{(2)}^{r}\left(X^{*}\right) \cong \mathbb{H}^{r}\left(X^{*}\right) & \text { if } r<n-1, \\ \mathbb{H}_{(2)}^{p, q}\left(X^{*}\right) \cong \mathbb{H}^{p, q}\left(X^{*}\right) & \text { if } p+q<n-1 .\end{cases}
$$

Since $\operatorname{dim} H^{\pi r}\left(X^{*}\right)$ and $\operatorname{dim} H^{p, q}\left(X^{*}\right)$ are finite on the above ranges (cf. [1]), by Serre's duality (4) is equivalent to that

$$
\begin{cases}\mathbb{H}_{(2)}^{r}\left(X^{*}\right) \cong \mathbb{H}_{0}^{r}\left(X^{*}\right) & \text { if } r>n+1 \\ H_{(2)}^{p, q}\left(X^{*}\right) \cong \mathbb{H}_{0}^{p, q}\left(X^{*}\right) & \text { if } p+q>n+1 .\end{cases}
$$

But (5) is immediate from Proposition 2.2 and Lemma 2.3. In fact, to show that the natural homomorphism from $H_{0}^{r}\left(X^{*}\right)$ to $H_{(2)}^{r}\left(X^{*}\right)$, say $\alpha$, is surjec- 
tive, one has only to know that square integrable forms on $X^{*}$ are in $L_{(2)}^{r}\left(X_{c}^{*}\right)$ around each singular point, is already assured for $r>n$ by Lemma 2.3. To show that $\alpha$ is injective, let $u$ be in $L_{(2)}^{r}\left(X^{*}\right)$ and compactly supported, such that there exists a $v \in L_{(2)}^{r-1}\left(X^{*}\right)$ with $d v=u$. Since $d v=0$ near the singularity, by the same reason as above one can replace $v$ by a compactly supported form in $L_{(2)}^{r-1}\left(X^{*}\right)$. The other isomorphism is proved similarly.

Remark It is also easy to prove (4) directly from Proposition 2.2 by using Lemma 2.4 instead of Lemma 2.3.

\section{\$4. Proof of Theorem 2}

Let $X$ be a compact $\mathbb{K}$ ähler space of pure dimension $n$. Now we need to distinguish two metrics on $X^{*}$, i.e. the original Kähler metric and a complete Kähler metric given in Proposition 3.1. Let us denote the original metric by $d s^{2}$ and make the distinction by $H_{(2)}^{r}\left(X^{*}\right)_{d s}^{2}$, etc.

While Theorem 1 was a consequence of Proposition 2.2, the proof of Theorem 2 is clearly reduced to the following local cohomology vanishing.

Proposition 4.1 For each singular point $\underline{\underline{ }} \in X$,

$$
\begin{cases}\underset{\lim _{\longrightarrow} H_{(2)}^{r}\left(X_{c}^{*}\right)_{d s^{2}}=0}{\longrightarrow} H_{(2)}^{p, q}\left(X_{c}^{*}\right)_{d s^{2}}=0 & \text { if } \quad r>n \\ \underset{\lim }{\longrightarrow} p+q>n\end{cases}
$$

and

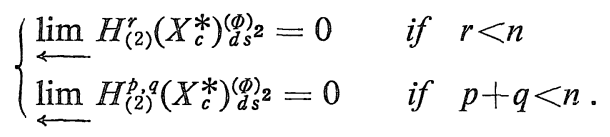

Here $H_{(2)}\left(X_{c}\right)_{d s^{2}}^{(\Phi)}$ denote the $L^{2}$ cohomologies with supports in $\Phi$ and the limits are taken by letting $c \rightarrow 0$.

Proof. We put $F_{\varepsilon}(z):=-\log \left(\left(c^{2}-|z|^{2}\right) \log ^{\varepsilon}(c /|z|)\right)$ for any $\varepsilon \geq 0$. Then $\partial \bar{\partial} F_{\varepsilon}>0$ on $X_{c}^{*}$ and $\partial \bar{\partial} F_{\varepsilon}$ converges to $-\partial \bar{\partial} \log \left(c^{2}-|z|^{2}\right)$ on the compact subsets of $X_{c}^{*}$.

We have

$$
\begin{aligned}
\partial \bar{\partial} F_{\varepsilon} & \\
= & \frac{\partial \bar{\partial}|z|^{2}}{c^{2}-|z|^{2}}+\frac{\partial|z|^{2} \bar{\partial}|z|^{2}}{\left(c^{2}-|z|^{2}\right)^{2}} \\
& +\varepsilon\left(\frac{\partial \bar{\partial} \log |z|}{\log (c /|z|)}+\frac{\partial \log |z| \bar{\partial} \log |z|}{\log ^{2}(c /|z|)}\right)
\end{aligned}
$$




$$
\begin{aligned}
\geq & \partial \log \left(c^{2}-|z|^{2}\right) \bar{\partial} \log \left(c^{2}-|z|^{2}\right) \\
& +\varepsilon^{-1} \partial \log \log ^{\varepsilon}(c /|z|) \bar{\partial} \log \log ^{\varepsilon}(c /|z|) .
\end{aligned}
$$

From the above inequality it is clear that $\partial \underline{e} F_{\varepsilon} \mid X_{c}$ is a complete $K_{\text {Ianhler metric }}$ on $X_{c}^{*}$ and $\left|\partial F_{\varepsilon}\right|_{\varepsilon} \leq 2$ if $0 \leq \varepsilon<1$. Here ||$_{\varepsilon}$ dejotes the length wich respect to $\partial \bar{\partial} \mathbb{F}_{\varepsilon}$.

Fron the above, the eigenvalues $\xi_{1}, \ldots, \xi_{N}$ of $\partial \bar{\partial} F_{\varepsilon}$ measured by the euclidean metric $\partial \bar{\partial}|z|^{2}$ are given by

$$
\begin{aligned}
& \xi_{j}=\frac{1}{c^{2}-|z|^{2}}+\frac{\varepsilon}{|z|^{2} \log (c /|z|)}, \quad 1 \leq j \leq N-\mathbb{1}, \\
& \xi_{N}=\frac{c^{2}}{\left(c^{2}-|z|^{2}\right)^{2}}+\frac{\varepsilon}{|z|^{2} \log ^{2}(c /|z|)} .
\end{aligned}
$$

Thus, similarly as in Lemma 2.3, one can find a consiant $A$ such that

$$
\|u\|_{\varepsilon} \leq A\|u\|_{d s^{2}} \text { for any } u \in \mathcal{L}_{(2)}^{r}\left(X_{c}^{*}\right)_{d s^{2}},
$$

if $0 \leq \varepsilon<1$. Here \|\|$_{\varepsilon}$ denoies the $\mathbb{L}^{2}$-norm with respect to $\partial \bar{\partial}_{\mathbb{F}_{\varepsilon}} \mid X_{c}^{*}$ 。 if $r>n$ and $d u=0$, then by Theorem 1.1, there exist ${ }_{\varepsilon} \in L_{(2)}^{\prime-1}\left(X_{c}^{*}\right)$ such that $d v_{\varepsilon}=u$ and $\left\|v_{\varepsilon}\right\|_{\varepsilon} \leq 4 A\|u\|_{d s^{2}}$ if $0<\varepsilon<1$. Lei $\left\{v_{\varepsilon}\right\}_{\nu=1}^{\infty}$ be a subsequence of

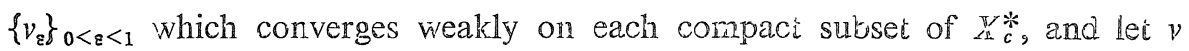
be the limit on $X_{c}^{*}$. Then $\|v\|_{0} \leq 4 A\|u\|_{d s^{2}}$ and $d v=u_{i}$ ince $\partial \bar{\partial}_{F_{0}}$ is quasiisometric near $\underline{o}$ to $d s^{2}$, this proves that $\lim w_{2}\left(\mathbb{X}_{c}^{*}\right)=0$ for $:>n$. The proofs of the other vanishings are similar except that for the vanishing vith supports in $\bar{D}$ one should use Lemma 2.4. This is a slight change and we shall not repeat the whole argument. The detail is leit to the reader.

\section{Reierences}

[1] Andreotti, A. and Grauert, Ti., Théorème de înitude pour la conomoicgie áes espaces complexes, Bull. Soc. Math. France, OD (1962), 193-259.

[2] Cheeger, J., Goresky, M. and IIacPherson, R., $\mathbb{E}^{2}$-cohomology and intersection homology of singular algebraic varieties, Seminar on Eiffercutial Geoneiry, Annals of Math. Studies 102, Princeton Univ. Press 1982.

[3] Deligne, P., Théorie de Hodge III, Pubi. R.H.E.S., Q4u (1972), j-77.

[4] Donnelly, H. and Fefferman, C., $L^{2}$-cohomology and inder iheorem for the Bergmar metric, Annals of Math., $\mathbb{1} \mathbb{1} \&$ (1983), 593-618.

[5] Fujiki, A., Hodge to de Rham spectral sequence on a sirongly pseudoconvex manifold (unpublished).

[6] Grauert, H., Charakierisierung der Holomorphiegebiete durcil dis vollsiändige Irähiersche Metrik, Math. Anı., 1.3i (1956), 38-75.

[7] Grauert, $\mathbb{H}$. and Riemenschneider, O., Kählersche f.rannigfaltiglreiten mil hyper$q$-konvexem Rand, Froblews in Analysis, Princeton Univ. Fress, 1970. 
[8] Hodge, W.V.D., The theory and applicaiion of harmonic integrals, Cambridge Univ. Press, London, 1952.

[9] Hörmander, L., An Introduction to Complex Analysis in Several Variables, NorthHolland, 1973.

[10] Hsiang, W.C. and Pati, V., $L^{2}$-cohomology of normal algebraic surfaces $\mathbb{I}$, Invent. math., 81 (1985), 395-412.

[11] Ohsawa, T., A reduction theorem for cohomology groups of very strongly $q$-convex Kähler manifolds, Invent. Math., 63 (1981), 335-354.

[12] Saper, L., $L^{2}$-cohomology and intersection homology of certain algebraic varieties with isolated singularities, Invent. math., 82 (1985), 207-255.

[13] Vesentini, E., Lectures on Levi convexity of complex manifolds and cohomology vanishing theorems, Tata Inst., Bombay, 1967.

[14] Wells, R.O., Differential analysis on complex manifolds, Prentice Hall, Englewood Cliffs, N.J., 1973. 